J. Herzog and B. Ulrich

Nagoya Math. J.

Vol. 120 (1990), 129-153

\title{
SELF-LINKED CURVE SINGULARITIES
}

\author{
JÜRGEN HERZOG* AND BERND ULRICH**
}

\section{Introduction}

Let $S$ be a three-dimensional regular local ring and let $I$ be a prime ideal in $S$ of height two. This paper is motivated by the question of when $I$ is a set-theoretic complete intersection and when the symbolic Rees algebra $S(I)=\oplus_{n \geq 0} I^{(n)} t^{n}$ is Noetherian. The connection between the two problems is given by a result of Cowsik which says that the Noetherian property of $S(I)$ implies that $I$ is a set-theoretic complete intersection ([1]).

The ideal $I$ is said to be linked to an $S$-ideal $J$ if there exists an $S$ regular sequence $\alpha_{1}, \alpha_{2}$ in $I \cap J$ such that $J=\left(\alpha_{1}, \alpha_{2}\right): I$ and $I=\left(\alpha_{1}, \alpha_{2}\right): J$, and $I$ is called self-linked if $I$ is linked to $I$ ([14]) (see also [15], [21], [22], where such ideals were studied). Of course every self-linked ideal is a set-theoretic complete intersection because $I^{2} \subset\left(\alpha_{1}, \alpha_{2}\right)$. As one of the main results in the first section of this paper, we prove that $I$ is selflinked in case $S / I$ has multiplicity at most five (Corollary 1.14). This follows from another result that gives a criterion in terms of the resolution, for when an almost complete intersection is self-linked (Theorem 1.1 and Proposition 1.8) (parts of the criterion are similar to results of Szpiro ([20]), Ferrand, Valla ([22]), Mohan Kumar). Using this criterion, we also characterize all self-linked monomial space curves (Corollary 1.10) and we show that normal almost complete intersections are self-linked (Corollary 1.9) (an ideal is called normal if all its powers are integrally closed). As an immediate consequence of Theorem 1.8, we also obtain Kumar's result that an ideal linked to a regular ideal is self-linked (Corollary 1.11).

In the second section we study the question of when $S(I)=S\left[I t, I^{(2)} t^{2}\right]$. Of course this equality forces $S(I)$ to be Noetherian. Here we will always assume that $I$ is an almost complete intersection in $S$ and that the ideal

\footnotetext{
Received November 14, 1988.

* Partially supported by DFG

** Partially supported by NSF
} 
generated by the entries of the relation matrix of $I$ is a complete intersection (these assumptions are automatically satisfied if $I$ is the defining ideal of a monomial curve ([5]) or if $I$ is a normal almost complete intersection ([25])). Relating the above question to the first section of the paper, we prove, as an analogue of Cowsik's result, that the equality $S(I)=S\left[I t, I^{(2)} t^{2}\right]$ forces $I$ to be self-linked (Corollary 2.5). This follows from our main result which gives a criterion for when $S(I)=S\left[I t, I^{(2)} t^{2}\right]$ (Theorem 2.1). The criterion is formulated in terms of the height of a determinantal ideal that can be easily computed from the presentation of $I$. To prove the theorem we explicitly describe a generator of the $S$-module $I^{(2)} / I^{2}$ (which is cyclic by [25]), and use this generator to obtain some information about a presentation of the algebra $S\left[I t, I^{(2)} t^{2}\right]$. Then the height condition in the theorem can be translated into a statement about the grade of a conductor ideal that tests the equality $S(I)=$ $S\left[I t, I^{(2)} t^{2}\right]$. Most of these ideas were introduced by W. Vasconcelos who also proved the above theorem in a more general context, but under the additional assumption that the Rees algebra $S[I t]=\oplus_{n \geq 0} I^{n} t^{n}$ is normal ([24]), [25]). We are grateful to him for providing these ideas and for his helpful comments concerning the material of our paper.

Theorem 2.1 yields a characterization of all monomial space curves for which the equality $S(I)=S\left[I t, I^{(2)} t^{2}\right]$ holds (Corollary 2.12) (extending results from [19]). We also determine when a self-linked ideal $I$ admits an element $w$ such that $w$ generates $I^{(2)} / I^{2}$ and is at the same time part of a regular sequence defining the self-linkage of $I$ (Corollary 2.10). It turns out that the existence of such an element implies the equality $S(I)=S\left[I t, I^{(2)} t^{2}\right]$ (Proposition 2.11).

Throughout this paper, $(S, m)$ will be a three-dimensional regular local ring, $I$ will be a prime ideal in $S$ of height two, $R=S / I$, and $\omega$ will be the canonical module of $R$. By ht, we will denote height of an ideal, $\mu$ will denote minimal number of generators, $r(R)=\mu(\omega)$ will be the type of $R$, and $e(R)$ will stand for the multiplicity of $R$. We will call an $n$ by $n+1$ matrix $A$ almost symmetric if the matrix obtained by deleting the last column of $A$ is symmetric, and by $I_{t}(A)$ we will denote the $S$ ideal generated by all $t$ by $t$ minors of $A$.

\section{$\S 1$. Self-linked space curve singularities}

We begin with a characterization of self-linked almost complete inter- 
sections. Parts of this theorem would also follow from [20], page 73, (the equivalence of a) and c)), [22] (the equivalence of a) and d)), or an unpublished result of Ferrand (the equivalence of a) and d)). However, we include a proof for the sake of completeness and because our proof is somewhat different.

THEOREM 1.1. In addition to the usual assumptions let $\mu(I)=3$. We consider a presentation $0 \rightarrow S^{2} \stackrel{A}{\rightarrow} S^{3} \rightarrow I \rightarrow 0$ where $A=\left(a_{i j}\right)$ is a 2 by 3 matrix with entries in $m$. then the following are equivalent.

a) $I$ is self-linked

b) $I^{2} \subset\left(\alpha_{1}, \alpha_{2}\right)$ for some elements $\alpha_{1}, \alpha_{2}$ in $m$

c) there exists an epimorphism $\varphi: I \rightarrow \omega$

d) there exists a 2 by 3 matrix $C=\left(c_{i j}\right)$ with entries in $S$ such that $I_{2}(C) \not \subset m$ and $\sum_{i=1}^{2} \sum_{j=1}^{3} a_{i j} c_{i j}=0$

e) there exists $a 3$ by 2 matrix $B$ with entries in $S$ such that $I_{2}(B)$ $\not \subset m$ and $A B$ is symmetric

f) there exists an invertible 3 by 3 matrix $D$ with entries in $S$ such that $A D$ is almost symmetric.

Proof. We first show that a) implies c). Let $\alpha_{1}, \alpha_{2}$ be an $S$-regular sequence with $I=\left(\alpha_{1}, \alpha_{2}\right): I$. By [14], $\omega \cong\left(\left(\alpha_{1}, \alpha_{2}\right): I\right) /\left(\alpha_{1}, \alpha_{2}\right)$, and hence $\omega \cong I /\left(\alpha_{1}, \alpha_{2}\right)$. Thus we may take $\varphi: I \rightarrow \omega$ to be the natural projection.

Next we prove that c) implies d). Write $-^{*}=\operatorname{Hom}_{S}(-, S)$. Then the exact sequence

$$
0 \longrightarrow S^{2} \stackrel{A}{\longrightarrow} S^{3} \longrightarrow S \longrightarrow R \longrightarrow 0
$$

yields an exact sequence

$$
0 \longrightarrow S^{*} \longrightarrow S^{3^{*}} \stackrel{A^{*}}{\longrightarrow} S^{2 *} \longrightarrow \omega \longrightarrow 0 .
$$

Therefore $\varphi$ induces a commutative diagram

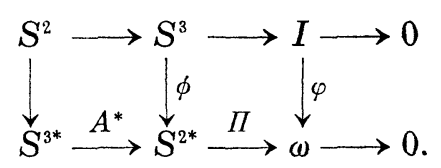

Denote the bases of $S^{2}$ and $S^{3}$ by $\left\{e_{1}, e_{2}\right\}$ and $\left\{h_{1}, h_{2}, h_{3}\right\}$ respectively, and let $w_{i}=\Pi\left(e_{i}^{*}\right)$. By Nakayama's Lemma, the surjectivity of $\varphi$ forces $\phi$ to be surjective, and hence there exists a new basis $\left\{g_{1}, g_{2}, g_{3}\right\}$ of $S^{3}$ such that $\phi\left(g_{1}\right)=e_{1}^{*}, \phi\left(g_{2}\right)=e_{2}^{*}, \phi\left(g_{3}\right)=0$, Let $E=\left(e_{i j}\right)$ be the invertible 3 by 3 
matrix with $\left(\begin{array}{l}h_{1} \\ h_{2} \\ h_{3}\end{array}\right)=E\left(\begin{array}{l}g_{1} \\ g_{2} \\ g_{3}\end{array}\right)$, and write $A E=\left(d_{i^{\prime}}\right)$. Because of the commutativity of the above diagram, it follows that

$$
0=\Pi\left(\phi\left(d_{11} g_{1}+d_{12} g_{2}+d_{13} g_{3}\right)\right)=d_{11} w_{1}+d_{12} w_{2} .
$$

On the other hand,

$$
0=\Pi\left(d_{11} e_{1}^{*}+d_{21} e_{2}^{*}\right)=d_{11} w_{1}+d_{21} w_{2} .
$$

Comparing the two equations we conclude that $d_{12} w_{2}=d_{21} w_{2}$. Since $\omega$ is isomorphic to an ideal in the domain $R$ and $w_{2} \neq 0$, it now follows that $d_{12} \equiv d_{21} \bmod I$. Note that $d_{12}-d_{21}=\sum_{j=1}^{3} a_{1 j} e_{j 2}-\sum_{j=1}^{2} a_{2 j} e_{j 1}$ and that $I=I_{2}(A) \subset I_{1}(A)$. Therefore $\sum_{j=1}^{3} a_{1 j} e_{j 2}-\sum_{j=1}^{3} a_{2 j} e_{j 1}$ can be written as $\sum_{i=1}^{2} \sum_{j=1}^{3} h_{i j} a_{i j}$ with $h_{i j} \in m$. Now define

$$
C=\left(c_{i j}\right)=\left(\begin{array}{rrr}
e_{12}-h_{11} & e_{22}-h_{12} & e_{32}-h_{13} \\
-e_{11}-h_{21} & -e_{21}-h_{22} & -e_{31}-h_{23}
\end{array}\right) .
$$

Then clearly $I_{2}(C) \not \subset m$ since $I_{3}(E) \not \subset m$, and $\sum_{i=1}^{2} \sum_{j=1}^{3} a_{i j} c_{i j}=0$.

We now prove that d) implies e). Simply define

$$
B=\left(\begin{array}{ll}
-c_{21} & c_{11} \\
-c_{22} & c_{12} \\
-c_{23} & c_{13}
\end{array}\right)
$$

Then $I_{2}(B) \not \subset m$, and $A B$ is symmetric since $\sum_{j=1}^{3} a_{1 j} c_{1 j}=\sum_{j=1}^{3} a_{2 j}\left(-c_{2 j}\right)$.

Next we see that e) implies $f$ ). Since $I_{2}(B) \not \subset m$, there exists an invertible 3 by 3 matrix $D$ such that

$$
D^{-1} B=\left(\begin{array}{ll}
1 & 0 \\
0 & 1 \\
0 & 0
\end{array}\right)
$$

Then

$$
A D=\left((A D)\left(D^{-1} B\right) \mid \begin{array}{l}
* \\
*
\end{array}\right)=\left(A B \mid \begin{array}{l}
* \\
*
\end{array}\right)
$$

where $A B$ is symmetric by assumption.

We now show that f) implies b). Since $I=I_{2}(A)=I_{2}(A D)$ we may assume that $A=\left(a_{i j}\right)$ is almost symmetric. Let $(-1)^{i+1} f_{i}$ be the maximal minor obtained from $A$ by deleting the $i$-th column, and set $f=a_{13} f_{1}+$ $a_{23} f_{2}$. Since $a_{21}=a_{12}$, it is easy to see that $f_{1}^{2}=-a_{23}^{2} f_{3}-a_{22} f, f_{1} f_{2}=a_{13} a_{23} f_{3}$ $+a_{12} f, f_{2}^{2}=-a_{13}^{2} f_{3}-a_{11} f$. Therefore $I^{2} \subset\left(f_{3}, f\right) \subset I$. 
Finally we prove that b) implies a). Since $I^{2} \subset\left(\alpha_{1}, \alpha_{2}\right) \subset I$ it follows that $\alpha_{1}, \alpha_{2}$ is an $S$-regular sequence in $I$. Moreover $I \subset\left(\alpha_{1}, \alpha_{2}\right): I$, and hence $I=\left(\alpha_{1}, \alpha_{2}\right): I$, because $I$ is prime.

Remark 1.2. The equivalence of d), e), f) in Theorem 1.1 and the proof that c) implies d) show that it suffices to check conditions d), e), or f) modulo the ideal $I$.

Remark 1.3. Assume that $\mu(I)=3$ and that $I$ is self-linked. Then by the equivalence of a) and $\mathrm{f}$ ) in Theorem 1.1, it follows that $I=I_{2}(A)$ where $A=\left(\begin{array}{lll}x & y & u \\ y & z & v\end{array}\right)$. Let $f_{i}$ denote the $i$-th maximal minor of $A$ with sign, then the proof that $f)$ implies a) shows that $I=\left(f_{3}, u f_{1}+v f_{2}\right): I$.

Remark 1.4. Assume that $I$ is self-linked with respect to the regular sequence $\alpha_{1}, \alpha_{2}$. Then after a permutation of indices if needed, $\alpha_{1} \notin m I$ and $\lambda \alpha_{1}+\alpha_{2} \in m I$ for some $\lambda \in S$.

Proof. It suffices to show that $\mu\left(I /\left(\alpha_{1}, \alpha_{2}\right)\right)=\mu(I)-1$. However, the minimal resolution

$$
0 \longrightarrow S^{n-1} \longrightarrow S^{n} \longrightarrow I \longrightarrow 0
$$

implies indeed that

$$
\begin{aligned}
\mu(I)-1 & =n-1=\mu(\omega)=\mu\left(\left(\left(\alpha_{1}, \alpha_{2}\right): l\right) /\left(\alpha_{1}, \alpha_{2}\right)\right) \\
& =\mu\left(I /\left(\alpha_{1}, \alpha_{2}\right)\right) .
\end{aligned}
$$

Notice that under the assumptions of Theorem 1.1, ht $I_{1}(A)=3$ and hence $\mu\left(I_{1}(A)\right) \geq 3$. Set $\bar{A}=\left(\bar{a}_{i j}\right)$ where $\bar{a}_{i j}$ denote the images of $a_{i j}$ in $R$. Then $I_{1}(\bar{A})=I_{1}(A) / I$ and $\mu\left(I_{1}(\bar{A})\right)=\mu\left(I_{1}(A)\right)$. The canonical module of $R$ can be identified with an $R$-ideal $\omega$, and then $\omega \omega^{-1}$ is an $R$-ideal that is independent of the choice of the canonical ideal $\omega$.

Remark 1.5. With the assumptions of Theorem 1.1, $I_{1}(\bar{A})=\omega \omega^{-1}$.

Proof. Since $\omega=\left(w_{1}, w_{2}\right)$ is a two-generated ideal in a domain, there is an exact sequence

$$
0 \longrightarrow \omega^{-1} \longrightarrow R^{2} \longrightarrow \omega \longrightarrow 0,
$$

where the image of $\omega^{-1}$ in $R^{2}$ is $\left\{\left(-w_{2} b, w_{1} b\right) \mid b \in \omega^{-1}\right\}$. Therefore the first Fitting ideal of $\omega$ as an $R$-module is $w_{2} \omega^{-1}+w_{1} \omega^{-1}=\omega \omega^{-1}$. On the other hand, we have a presentation 


$$
R^{3^{*}} \stackrel{\bar{A}^{*}}{\longrightarrow} R^{2^{*}} \longrightarrow \omega \longrightarrow 0
$$

Using this presentation to compute $F_{1}$, we obtain $F_{1}=I_{1}\left(\bar{A}^{*}\right)=I_{1}(\bar{A})$. Therefore $I_{1}(\bar{A})=\omega \omega^{-1}$.

Concerning the ideal $I_{1}(A)$ or $I_{1}(\bar{A})$, we will often make the following

Assumption 1.6. Suppose $\mu(I)=3$, let $A$ be a 2 by 3 matrix with entries in $m$ such that $I=I_{2}(A)$, and assume that $\mu\left(I_{1}(A)\right)=3$ or, equivalently, that $I_{1}(A)$ is a complete intersection in $S$.

Notice that this assumption is automatically satisfied if $I$ is the defining ideal of a monomial space curve ([5]), or if $I$ is a normal almost complete intersection ([23], [25]).

To apply Theorem 1.1 we first need a lemma.

Lemma 1.7. Under the Assumption 1.6 exactly one of the following two conditions holds:

a) there exists an invertible 3 by 3 matrix $D$ with entries in $S$ such that for $A D=\left(e_{i j}\right), e_{11}, e_{21}$ form part of a minimal system of generators of $I_{1}(A D)=I_{1}(A)$

b) there exists an invertible 2 by 2 matrix $E$ with entries in $S$ such that for $E A=\left(d_{i j}\right),\left(d_{21}, d_{22}, d_{23}\right) \subset m\left(d_{11}, d_{12}, d_{13}\right)$.

Proof. First note that we may perform elementary row and column operations on $A$. As for column operations, this is part of the definition in a), and for row operations, this is part of the definition in b). To see that a) is preserved under elementary row operations, simply notice that the vector space dimension of each column as a subspace of $I_{1}(A) / m I_{1}(A)$ is invariant under such operations. Finally, to show that $b$ ) is not changed by elementary column operations, we remark that by Nakayama's Lemma, b) is equivalent to the inclusion $\left(d_{21}, d_{22}, d_{23}\right) \subset m I_{1}(A)$, which is clearly preserved under column operations.

If one of the columns of $A$ is part of a minimal generating set of $I_{1}(A)$, then a) is trivially satisfied. Therefore we may assume that $a_{11}, a_{12}$, but none of the columns of $A$ form part of a generating set of $I_{1}(A)$. Then $a_{21} \in\left(a_{11}\right)+m I_{1}(A)$ and $a_{22} \in\left(a_{12}\right)+m I_{1}(A)$. Write $a_{21} \equiv \lambda_{1} a_{11} \bmod m I_{1}(A)$ and $a_{22} \equiv \lambda_{2} a_{12} \bmod m I_{1}(A)$. If $\lambda_{1} \not \equiv \lambda_{2} \bmod m$, then adding the second column to the first column yields a matrix which satisfies condition a).

If however $\lambda_{1} \equiv \lambda_{2} \bmod m$, we may subtract $\lambda_{1}$ times the first row from the second row to assume that $a_{21} \in m I_{1}(A)$ and $a_{22} \in m I_{1}(A)$. Then 
$I_{1}(A) /\left(a_{11}, a_{12}\right)$ can be generated by $a_{13}$ or $a_{23}$. Since $a_{21}$ and $a_{22}$ are in $m I_{1}(A)$, we may add a suitable multiple of the second row to the first row to assume that $I_{1}(A)=\left(a_{11}, a_{12}, a_{13}\right)$. Again we may suppose that $a_{23} \in\left(a_{13}\right)$ $+m I_{1}(A)$. As above, if $a_{23} \notin m I_{1}(A)$, then adding the third column to the first column yields a matrix satisfying condition a) (note that $a_{21} \in m I_{1}(A)$ ). However, if $a_{23} \in m I_{1}(A)$, then $\left(a_{21}, a_{22}, a_{23}\right) \subset m I_{1}(A)$, and hence $I_{1}(A)=$ $\left(a_{11}, a_{12}, a_{13}\right)$. Therefore $\left(a_{21}, a_{22}, a_{23}\right) \subset m\left(a_{11}, a_{12}, a_{13}\right)$, which is condition b).

Thus we have shown that either a) or b) hold true. Moreover it is clear from the remarks at the beginning of the proof that these conditions are mutually exclusive.

Now let $I$ and $A=\left(a_{i j}\right)$ be as in Theorem 1.1, let $\underline{x}=x_{1}, x_{2}, x_{3}$ be a sequence of elements in $m$ such that $I_{1}(A) \subset\left(x_{1}, x_{2}, x_{3}\right)$, and write $a_{i j}=$ $\sum_{k=1}^{3} a_{i j}^{(k)} x_{k}$. Since $I$ is of linear type ([8]), the Rees-algebra $S[I t]$ has a presentation $S\left[T_{1}, T_{2}, T_{3}\right] /\left(F_{1}, F_{2}\right)$, where $T_{1}, T_{2}, T_{3}$ are indeterminates over $S$, and

$$
F_{i}=\sum_{j=1}^{3} a_{i j} T_{j}=\sum_{j=1}^{3}\left(\sum_{j=1}^{3} a_{i \jmath}^{(k)} x_{k}\right) T_{j}=\sum_{j=1}^{3}\left(\sum_{j=1}^{3} a_{i j}^{(k)} T_{j}\right) x_{k}=\sum_{k=1}^{3} \gamma_{i k} x_{k},
$$

with

$$
\gamma_{i k}=\sum_{j=1}^{3} a_{i j}^{(k)} T_{j}
$$

Now it seems natural to consider the matrix

$$
\Gamma=\left(\begin{array}{lll}
\gamma_{11} & \gamma_{12} & \gamma_{13} \\
\gamma_{21} & \gamma_{22} & \gamma_{23}
\end{array}\right)
$$

and the ideal $\Delta^{x}=I_{2}(\Gamma) \subset S\left[T_{1}, T_{2}, T_{3}\right]$. Finally let $\bar{\Gamma}$ be the matrix obtained by reducing $\Gamma$ modulo $m$ and let $\bar{\Delta}^{x}=I_{2}(\bar{\Gamma})$ be the image of $\Delta^{x}$ in $S / m\left[T_{1}, T_{2}, T_{3}\right]$. One can show that $\bar{\Delta}^{x}$ only depends on $I$ and the ideal $\left(x_{1}, x_{2}, x_{3}\right)$, being independent of the choice of $A$ and $\underline{x}=x_{1}, x_{2}, x_{3}$. The ideals $\bar{\Delta}^{x}$ were first introduced by W. Vasconcelos ([23]) who showed that $S[I t]$ is normal if and only if $\bar{\Delta}^{x} \neq 0$ for a regular system of parameters $\underline{x}$ in $S$. We will mostly consider the case where $\mu\left(I_{1}(A)\right)=3$. We then take $\underline{x}=x_{1}, x_{2}, x_{3}$ to be a system of generators of $I_{1}(A)$, and simply write $\bar{\Delta}$ instead of $\bar{\Delta}^{x}$. We are now ready to give another characterization of self-linked ideals. Part of our proposition (the fact that c) implies a)) could also be concluded from the work of Mohan Kumar.

Proposition 1.8. Under the Assumption 1.6 the following are equi- 
valent:

a) I is self-linked

b) $\bar{\Delta} \neq 0$

c) A satisfies 1.7 a).

Proof. By Lemma 1.7, $A$ satisfies exactly one of the conditions 1.7 a) or $1.7 \mathrm{~b}$ ). Thus it suffices to show that if $1.7 \mathrm{a}$ ) holds then $I$ is selflinked and $\bar{\Delta} \neq 0$, whereas if $1.7 \mathrm{~b}$ ) holds then $I$ is not self-linked and $\bar{\Delta}=0$.

So assume 1.7 a). Replacing $A$ by $A D$ we may suppose that

$$
A=\left(\begin{array}{lll}
x_{1} & x_{2} & a_{13} \\
x_{3} & a_{22} & a_{23}
\end{array}\right)
$$

where $I_{1}(A)=\left(x_{1}, x_{2}, x_{3}\right)$. Thus there are elements $c_{11}, c_{21}, c_{12}$ in $S$ such that $a_{13}+a_{22}=-c_{11} x_{1}-c_{12} x_{2}-c_{21} x_{3}$. Now the matrix

$$
C=\left(\begin{array}{lll}
c_{11} & c_{12} & 1 \\
c_{21} & 1 & 0
\end{array}\right)
$$

satisfies condition d) in Theorem 1.1, and hence $I$ is self-linked by Theorem 1.1. Moreover,

$$
\Gamma=\left(\begin{array}{ccc}
T_{1}+a_{13}^{(1)} T_{3} & * & a_{13}^{(3)} T_{3} \\
* & * & T_{1}+a_{22}^{(3)} T_{2}+a_{23}^{(3)} T_{3}
\end{array}\right)
$$

and hence $\bar{\Delta} \neq 0$.

Now assume $1.7 \mathrm{~b})$. Replacing $A$ by $E A$ we are in the situation where

$$
A=\left(\begin{array}{lll}
x_{1} & x_{2} & x_{3} \\
a_{21} & a_{22} & a_{23}
\end{array}\right)
$$

with $\left(a_{21}, a_{22}, a_{23}\right) \subset m\left(x_{1}, x_{2}, x_{3}\right)$. Now let $C=\left(c_{i j}\right)$ be a 2 by 3 matrix with entries in $S$ such that $\sum_{i=1}^{2} \sum_{j=1}^{3} a_{i j} c_{i j}=0$. Then $x_{1} c_{11}+x_{2} c_{12}+x_{3} c_{13} \equiv$ $0 \bmod m$, and therefore $\left(c_{11}, c_{12}, c_{13}\right) \subset m$. Thus condition $\left.\mathrm{d}\right)$ in Theorem 1.1 is violated and $I$ cannot be self-linked by Theorem 1.1. Moreover,

$$
\bar{\Gamma}=\left(\begin{array}{ccc}
T_{1} & T_{2} & T_{3} \\
0 & 0 & 0
\end{array}\right)
$$

and hence $\bar{\Delta}=0$.

Corollary 1.9. Assume that $\mu(I)=3$ and $I$ is normal. Then $I$ is self-linked. 
Proof. Since $S[I t]$ is normal it follows from [23] and [25] that for a suitable regular system of parameters $\underline{x}=x_{1}, x_{2}, x_{3}$ in $S, \bar{J}^{x} \neq 0$ and $I_{1}(A)$ $=\left(x_{1}, x_{2}, x_{3}^{c}\right)$ where $c$ is some positive integer. In particular Assumption 1.6 is satisfied. Moreover $\Delta^{x} \subset \Delta^{x_{1}, x_{2}, x_{3}^{c}}=\Delta$, and hence $\bar{\Delta} \neq 0$. Now Proposition 1.8 implies that $I$ is self-linked.

Corollary 1.10. Let $k$ be a field, $S=k \llbracket X, Y, Z \rrbracket$ a power series ring, and $R=S / I=k \llbracket t^{n_{1}}, t^{n_{2}}, t^{n_{3}} \rrbracket$ a monomial space curve singularity that is not a complete intersection. Write $I=I_{2}(A)$ with

$$
A=\left(\begin{array}{lll}
X^{\alpha_{1}} & Y^{\beta_{1}} & Z^{\gamma_{1}} \\
Z^{\gamma_{2}} & X^{\alpha_{2}} & Y^{\beta_{2}}
\end{array}\right)
$$

(this is always possible by [5]). Then the following are equivalent:

a) $I$ is not self-linked

b) $\alpha_{1}<\alpha_{2}$ and $\beta_{1}<\beta_{2}$ and $\gamma_{1}<\gamma_{2}$, or $\alpha_{1}>\alpha_{2}$ and $\beta_{1}>\beta_{2}$ and $\gamma_{1}>\gamma_{2}$.

Proof. First notice that Assumption 1.6 is satisfied.

Assume a) and suppose that b) does not hold. Then after a cyclic permutation of the variables $X, Y, Z$, either $\alpha_{1} \geq \alpha_{2}$ and $\beta_{1} \leq \beta_{2}$ or $\alpha_{1} \leq \alpha_{2}$ and $\beta_{1} \geq \beta_{2}$. In the first case, $a_{12}, a_{22}$ form part of a minimal generators of $I_{1}(A)$. In the second case, $a_{11}, a_{21}$ or $a_{13}, a_{23}$ form part of a minimal system of generators of $I_{1}(A)$ depending on whether $\gamma_{1} \geq \gamma_{2}$ or $\gamma_{1} \leq \gamma_{2}$. In any case, Proposition 1.8 would imply that $I$ is self-linked.

Next assume that b) holds. Then condition $1.7 \mathrm{~b}$ ) is satisfied, and hence by Lemma 1.7 and Proposition 1.8, $I$ is not self-linked.

From Proposition 1.8 we also obtain a result of Mohan Kumar which has inspired part of our work.

Corollary 1.11 (Mohan-Kumar). Assume that $I$ is linked to a regular ideal. Then $I$ is self-linked.

Proof. Suppose that $I$ is linked to a regular ideal $\left(x_{1}, x_{2}\right)$ with respect to the regular sequence $f_{1}, f_{2}$. Then $I /\left(f_{1}, f_{2}\right)$ is generated by one element $f([14])$, and we may assume that $f, f_{1}, f_{2}$ minimally generate $I$. Since $\left(x_{1}, x_{2}\right) f \subset\left(f_{1}, f_{2}\right)$, we obtain equations $x_{i} f+a_{i 2} f_{1}+a_{i 3} f_{2}=0$ for $1 \leq i \leq 2$. Because $x_{1}, x_{2}$ form part of a regular system of parameters of $S$ it follows that both relations form part of a minimal generating set of the first syzygy module of $I$. Thus we may take 


$$
A=\left(\begin{array}{lll}
x_{1} & a_{12} & a_{13} \\
x_{2} & a_{22} & a_{23}
\end{array}\right)
$$

Since $I_{1}(A) /\left(x_{1}, x_{2}\right)$ is an ideal in the discrete valuation domain $S /\left(x_{1}, x_{2}\right)$, we conclude that $I_{1}(A) /\left(x_{1}, x_{2}\right)$ is cyclic. Now the claim follows from Proposition 1.8 .

Corollary 1.12. In addition to Assumption 1.6 suppose that $I \not \subset m^{3}$. Then $I$ is self-linked.

Proof. Suppose that $I$ is not self-linked. Then by Proposition 1.8 and Lemma 1.7, we could assume that $\left(a_{21}, a_{22}, a_{23}\right) \subset m\left(a_{11}, a_{12}, a_{13}\right)$. But then $I=I_{2}(A) \subset m\left(I_{1}(A)\right)^{2} \subset m^{3}$.

For our next result we first need a lemma.

LemMA 1.13. Let $S^{\prime}$ be a faithfully flat extension of $S$ such that $\left(S^{\prime}, m^{\prime}\right)$ is a three-dimensional regular local ring and $I^{\prime}=I S$ is a prime ideal. Then the following are equivalent:

a) I satisfies Assumption 1.6 and is self-linked in $S$

b) $I^{\prime}$ satisfies Assumption 1.6 and is self-linked in $S^{\prime}$.

Proof. Clearly $I$ satisfies 1.6 if and only if $I^{\prime}$ does. Now consider $\bar{\Delta} \subset S / m\left[T_{1}, T_{2}, T_{3}\right]=Q$, and the corresponding ideal of $I^{\prime}, \bar{d}^{\prime} \subset S^{\prime} / m^{\prime}\left[T_{1}\right.$, $\left.T_{2}, T_{3}\right]=Q^{\prime}$. Then $Q \subset Q^{\prime}$ and $\bar{\Delta} Q^{\prime}=\bar{\Delta}^{\prime}$. Now the claim follows from Proposition 1.8 .

Corollary 1.14. Assume that $e(R) \leq 5$. Then $I$ is self-linked.

Proof. By Lemma 1.13 we may assume that $R$ has infinite residue class field. Then there exists an element $z$ in $S$ with $z \in m \backslash m^{2}$ such that $z$ is $R$-regular and $e(R /(z))=e(R)$. By "e-" we denote reduction modulo $z$. Then $\bar{R}=\bar{S} / \bar{I}$, and we consider the associated graded ring of $\bar{R}$, $\operatorname{gr}_{m}(\bar{R})=\operatorname{gr}_{m}(\bar{S}) / J=Q / J$ where $Q=k[X, Y]$ and $k=\bar{S} / \bar{m}$. We will prove the following:

(1.15) If $J$ is not a complete intersection then $J=\left(F_{1}, F_{2}, F_{3}\right)$ where $F_{1}, F_{2}, F_{3}$ are homogeneous elements in $Q$, and there are two homogeneous relations $\sum_{j=1}^{3} B_{i j} F_{j}=0,1 \leq i \leq 2$, such that at least two of the elements $B_{i j}(1 \leq i \leq 2,1 \leq j \leq 3)$ are linearly independent linear forms in $Q$.

We first show how Corollary 1.14 follows from (1.15). If $J$ is a complete intersection, then $\bar{I}$ and $I$ are complete intersections. Thus we may 
assume that $J$ is not a complete intersection. Let $f_{i}$ be elements in $I$ such that the leading forms of $\bar{f}_{i}$ are $F_{i}$. Then $\bar{I}=\left(\bar{f}_{1}, \bar{f}_{2}, \bar{f}_{3}\right)$ and hence $I=\left(f_{1}, f_{2}, f_{3}\right)$. In particular, $\mu(I)=3$. Also $\bar{f}_{1}, \bar{f}_{2}, \bar{f}_{3}$ form a standard basis of $\bar{I}$ and hence by [17], we can find relations in $\bar{S}, \sum_{j=1}^{3} \bar{b}_{i j} \bar{f}_{j}=0,1 \leq i$ $\leq 2$, such that $B_{i j}$ are the leading forms of $\bar{b}_{i j}$. Then $\sum_{j=1}^{3} b_{i j} f_{j} \in(z) \cap I=z I$, and changing $a_{i j}$ modulo the ideal $(z)$ we obtain relations in $S, \sum_{j=1}^{3} b_{i j} f_{j}$ $=0,1 \leq i \leq 2$, such that $B_{i j}$ are the leading forms $\bar{b}_{i j}$. Therefore by assumption on the elements $B_{i j}$, at least two of the elements $b_{i j}$ form part of a regular system of parameters in $S$. Call these two elements $x_{1}, x_{2}$, and let $A$ be the full relation matrix of $I$. Then $\left(x_{1}, x_{2}\right) \subset I_{1}(A)$ and $I_{1}(A) /\left(x_{1}, x_{2}\right)$ is cyclic. Thus $\mu\left(I_{1}(A)\right)=3$ and hence Assumption 1.6 is satisfied. Moreover $I \not \subset m^{3}$ since otherwise $e(R) \geq 6$. Now Corollary 1.14 follows from Corollary 1.12.

To prove (1.15), we first list all possibilities for two linearly independent quadratic forms $h_{1}, h_{2}$ in the polynomial ring $Q=k[X, Y]$. Write $h_{1}=\alpha_{11} x^{2}+\alpha_{12} x y+\alpha_{13} y^{2}, h_{2}=\alpha_{21} x^{2}+\alpha_{22} x y+\alpha_{23} y^{2}$. We may assume that the matrix $\left(\alpha_{i j}\right)$ is either

$$
\left(\begin{array}{lll}
1 & 0 & \alpha_{13} \\
0 & 1 & \alpha_{23}
\end{array}\right) \quad \text { or } \quad\left(\begin{array}{lll}
1 & \alpha_{12} & 0 \\
0 & \alpha_{22} & 1
\end{array}\right)
$$

In the second case we may even assume that $\alpha_{12}=\alpha_{22}=0$, since otherwise, this case reduces to the first case. Therefore $h_{1}=X^{2}+\alpha_{13} Y^{2}, h_{2}=X Y$ $+\alpha_{23} Y^{2}$, or $h_{2}=X^{2}, h_{2}=Y^{2}$. In the first case, we may replace $X$ by $X^{\prime}=X+\alpha_{23} Y$ to obtain $h_{2}=X^{\prime} Y$. After adding a suitable multiple of $h_{2}$ to $h_{1}$ and writing $X$ instead of $X^{\prime}$, we may assume that $h_{1}=X^{2}+\alpha Y^{2}$, $h_{2}=X Y$, or in the second case $h_{1}=X^{2}, h_{2}=Y^{2}$.

Now we are ready to prove (1.15). Notice that $e(Q / J) \leq 5$ since $e(Q / J)=e(R)$, and $J \subset\left(X^{2}, X Y, Y^{2}\right)$ since $J$ is not a complete intersection.

If $e(Q / J)=3$, then $J=\left(X^{2}, X Y, Y^{2}\right)$ and (1.15) is clearly true.

So assume that $e(Q / J)=4$. Then the Hilbert series of $Q / J$ is $1+2 t$ $+t^{2}$. Therefore $J=\left(h_{1}, h_{2}\right)+(X, Y)^{3}$, where $h_{1}, h_{2}$ are two linearly independent quadratic forms in $Q$. Hence by the above, $h_{1}=X^{2}+\alpha Y^{2}, h_{2}=$ $X Y$, or $h_{1}=X^{2}, h_{2}=Y^{2}$. If $h_{1}=X^{2}+\alpha Y^{2}$ with $\alpha \neq 0, h_{2}=X Y$, or if $h_{1}=X^{2}, h_{2}=Y^{2}$, then $J=\left(h_{1}, h_{2}\right)$ would be a complete intersection. Thus we may assume that $h_{1}=X^{2}, h_{2}=X Y$. Then $J=\left(X^{2}, X Y, Y^{3}\right)$, and $Y\left(X^{2}\right)$ $-X(X Y)=0$ is the desired relation in (1.15).

Finally let $e(Q / J)=5$. Then the Hilbert series of $Q / J$ is $1+2 t+t^{2}$ 
$+t^{3}$ or $1+2 t+2 t^{2}$. In the first case $J$ contains two linearly independent quadratic forms $h_{1}, h_{2}$. If $h_{1}=X^{2}+\alpha Y^{2}$ with $\alpha \neq 0, h_{2}=X Y$, or if $h_{1}=X^{2}$, $h_{2}=Y^{2}$, then $(X, Y)^{3} \subset J$, and hence $e(Q / J)<5$. Therefore we may assume that $h_{1}=X^{2}, h_{2}=X Y$, and from the Hilbert series we obtain that $J=$ $\left(X^{2}, X Y\right)+(X, Y)^{4}=\left(X^{2}, X Y, Y^{4}\right)$. Now again $Y\left(X^{2}\right)-X(X Y)$ is the desired relation in (1.15). Finally consider the second case where the Hilbert series is $1+2 t+2 t^{2}$. Then either $J=\left(X^{2}+\alpha X Y+\beta Y^{2}\right)+(X, Y)^{3}=$ $\left(X^{2}+\alpha X Y+\beta Y^{2}, X Y^{2}, Y^{3}\right)$ and $Y\left(X Y^{2}\right)-X\left(Y^{3}\right)=0$ is the desired relation in (1.15), or else $J=(X Y)+(X, Y)^{3}=\left(X Y, X^{3}, Y^{3}\right)$ and $X^{2}(X Y)-$ $Y\left(X^{3}\right)=0, Y^{2}(X Y)-X\left(Y^{3}\right)=0$ are the desired relation in (1.15).

\section{§2. Symbolic Rees algebras of space curve singularities}

Recall that the $n$-th symbolic power of $I$ is defined as $I^{(n)}=S \cap I^{n} S_{I}$ and that the symbolic Rees algebra of $I$ is $S(I)=\oplus_{n \geq 0} I^{(n)} t^{n}$ (viewed as a subring of the polynomial ring $S[t])$. This algebra contains the Rees algebra $S[I t]$, but equality only holds if $I$ is a complete intersection ([2]). With the methods of Vasconcelos ([25]) we want to study the question of when $S(I)=S\left[I t, I^{(2)} t^{2}\right]$. As $S(I)$ need not even be Noetherian ([16]), this is a rather strict requirement on $I$.

For the remainder of this paper we will suppose that $I$ satisfies Assumption 1.6. We will often make use of the ideal $\bar{\Delta} \subset S / m\left[T_{1}, T_{2}, T_{3}\right]$ which was defined prior to Proposition 1.8. Notice that ht $\bar{\Delta} \leq 2$ since $\bar{\Delta}=I_{2}(\bar{\Gamma})$, and ht $\bar{\Delta} \geq 1$ if and only if $I$ is self-linked (Proposition 1.8).

The next theorem answers the above question.

TheOREM 2.1. Under the Assumption 1.6 the following are equivalent:

a) $S(I)=S\left[I t, I^{(2)} t^{2}\right]$

b) ht $\bar{\Delta}=2$.

This theorem has been proved by W. Vasconcelos in a more general context, but with the additional assumption that $S[I t]$ is normal ([25]) (his proof also applies to the case where $\bar{\Delta} \neq 0$ ). For different results concerning the Noetherian property of symbolic Rees algebras, we also refer to the work of S. Eliahou ([3]), C. Huneke ([9], [11]), M. Morales ([13]), and P. Schenzel ([18], [19]). As a first step in the proof of Theorem 2.1, we describe the second symbolic power $I^{(2)}$. This is done in Proposition 2.2, which follows again from Vasconcelos in case $S[I t]$ is normal (or also from unpublished computations by Huneke). In particular it will 
turn out that $I^{(2)} / I^{2}$ is cyclic, a fact which has been shown for monomial space curves in [3], [10], and [19].

We first have to fix some more notation. Let $(-1)^{i+1} f_{i}, 1 \leq i \leq 3$, be the maximal minor of the 2 by 3 matrix $A=\left(a_{i j}\right)$ obtained by deleting the $i$-th column, so that $I=I_{2}(A)=\left(f_{1}, f_{2}, f_{3}\right)$, write $I_{1}(A)=\left(x_{1}, x_{2}, x_{3}\right)$, let $\Gamma=\left(\gamma_{i j}\right)$ be the 2 by 3 matrix defined prior to Proposition 1.8, write $P$ for the polynomial ring $S\left[T_{1}, T_{2}, T_{3}\right]$ and $B$ for the Rees algebra $S[I t]$, and in $P$ consider $F_{1}, F_{2}$ with

$$
\left(\begin{array}{l}
F_{1} \\
F_{2}
\end{array}\right)=A\left(\begin{array}{l}
T_{1} \\
T_{2} \\
T_{3}
\end{array}\right)=\Gamma\left(\begin{array}{l}
x_{1} \\
x_{2} \\
x_{3}
\end{array}\right) .
$$

Then $\left(F_{1}, F_{2}\right) P=\operatorname{ker} \varphi$ where $\varphi: P \rightarrow B$ is the natural projection, mapping $T_{i}$ to $f_{i} t$. Finally let $(-1)^{i+1} \Delta_{i} \quad 1 \leq i \leq 3$, be the maximal minor of $\Gamma$ obtained by deleting the $i$-th column, and let $(-1)^{i+1} D_{i}, 1 \leq i \leq 3$, be the $i$-th minor of the 2 by 3 matrix we get from if we replace $T_{j}$ by $f_{j}$. Notice that $\varphi\left(\Delta_{i}\right)=D_{i} t^{2}$.

Under the Assumption 1.6 we now explicitly describe $I$. Huneke and Vasconcelos have shown that $\operatorname{Ext}_{R}^{1}\left(I^{(2)} / I^{2}, \omega\right) \cong S / I_{1}(A)([10],[25])$, and by local duality, $I_{1}(A)$ also annihilates $I^{(2)} / I^{2}$, or in other words, $I^{(2)} \subset I^{2}: I_{1}(A)$. As $I_{1}(A) \not \subset I$, we always have that $I^{2}: I_{1}(A) \subset I^{(2)}$, and therefore $I^{(2)}=$ $I^{2}: I_{1}(A)$.

Let $K$ be the quotient field of $S$, and let $x \in I_{1}(A), x \neq 0$. Then $I^{(2)}=$ $I^{2}: I_{1}(A)=I^{2}: I_{1}(A)$ since grade $I_{1}(A)>1$, and therefore $x I^{(2)}=x I^{2}: I_{1}(A)$ $=x I^{2}: I_{1}(A)$. Now consider $B=\oplus_{n \geq 0} I^{n} t^{n} \subset S[t]$ and read the above equation in $S[t]$. Then $x I^{(2)} t^{2}=\left[x B \underset{S[t]}{:} I_{1}(A)\right]_{2}$, the degree two part of $x B \underset{S[t]}{:} I_{1}(A)$. But $x B \underset{S[t]}{:} I_{1}(A)=x B \underset{S[t]}{:} I_{1}(A) B=x B: I_{B}(A) B$ since $x B \subset I_{1}(A) B$. Thus $x I^{(2)} t^{2}=\left[x B: I_{B}(A) B\right]_{2}$, where $x B:_{B} I_{1}(A) B$ is a $B$-ideal that is linked to $I_{1}(A) B$ with respect to the regular element $x$.

Now we use our assumption $I_{1}(A)=\left(x_{1}, x_{2}, x_{3}\right)$ and choose $x=x_{1}$. We will prove that $x_{1} B:\left(x_{1}, x_{2}, x_{3}\right) B=\left(x_{1}, D_{1} t^{2}\right) B$. Notice that $B=P /\left(F_{1}, F_{2}\right)$, and that $F_{1}, F_{2}, x_{1}$ is a regular sequence in $P=S\left[T_{1}, T_{2}, T_{3}\right]$. Hence by homogeneity, also $x_{1}, F_{1}, F_{2}$ is a $P$-regular sequence. Thus if "/" denotes reduction modulo $x_{1} P$, then $F_{1}^{\prime}=\gamma_{12}^{\prime} x_{2}^{\prime}+\gamma_{13}^{\prime} x_{3}^{\prime}, F_{2}^{\prime}=\gamma_{22}^{\prime} x_{2}^{\prime}+\gamma_{23}^{\prime} x_{3}^{\prime}$ form a $P^{\prime}$. regular sequence contained in the complete intersection $\left(x_{2}^{\prime}, x_{3}^{\prime}\right) P^{\prime}$. But then it is well known that 


$$
\left(F_{1}^{\prime}, F_{2}^{\prime}\right) P^{\prime}:\left(x_{2}^{\prime}, x_{3}^{\prime}\right) P^{\prime}=\left(F_{1}^{\prime}, F_{2}^{\prime}, \operatorname{det}\left(\begin{array}{ll}
\gamma_{12}^{\prime} & \gamma_{13}^{\prime} \\
\gamma_{22}^{\prime} & \gamma_{23}^{\prime}
\end{array}\right)\right) P^{\prime}=\left(F_{1}^{\prime}, F_{2}^{\prime}, \Delta_{1}^{\prime}\right) P^{\prime} .
$$

Therefore

$$
\begin{aligned}
\left(F_{1}, F_{2}, x_{1}\right) P:\left(x_{1}, x_{2}, x_{3}\right) P & =\left(x_{1}, F_{1}, F_{2}\right) P:\left(x_{1}, x_{2}, x_{3}\right) P \\
& =\left(x_{1}, F_{1}, F_{2}, \Delta_{1}\right) P=\left(F_{1}, F_{2}, x_{1}, \Delta_{1}\right) P .
\end{aligned}
$$

and applying the epimorphism $\varphi$ we obtain that

$$
x_{1} B:\left(x_{1}, x_{2}, x_{3}\right) B=\left(x_{1}, D_{1} t^{2}\right) B .
$$

Therefore

$$
x_{1} I^{(2)} t^{2}=\left[x_{1} B: \underset{B}{:}\left(x_{1}, x_{2}, x_{3}\right) B\right]_{2}=\left(x_{1} I^{2}, D_{1}\right) t^{2},
$$

and hence $I^{(2)}=\left(I^{2}, D_{1} / x_{1}\right)$.

Finally notice that over $S, \Gamma\left(\begin{array}{l}x_{1} \\ x_{2} \\ x_{3}\end{array}\right)=\left(\begin{array}{l}F_{1} \\ F_{2}\end{array}\right)$ and $\Gamma\left(\begin{array}{l}\Delta_{1} \\ \Delta_{2} \\ \Delta_{3}\end{array}\right)=0$. Now apply $\varphi$ and write $\varphi(\Gamma)=C$. Then over $B, C\left(\begin{array}{l}x_{1} \\ x_{2} \\ x_{3}\end{array}\right)=0$ and $C\left(\begin{array}{l}D_{1} t^{2} \\ D_{2} t^{2} \\ D_{3} t^{3}\end{array}\right)=0$. Since $I_{2}(C)=\left(D_{1}, D_{2}, D_{3}\right) t^{2} B \neq 0, C$ has rank two and hence $D_{1} / x_{1}=D_{2} / x_{2}=D_{3} / x_{3}$.

We summarize our computations in the following proposition:

Proposifion 2.2. Suppose that Assumption 1.6 is satisfied and write $w=D_{1} / x_{1}$. Then $w=D_{1} / x_{1}=D_{2} / x_{2}=D_{3} / x_{3}$, and $I^{(2)}=\left(I^{2}, w\right)$.

Before we come to the proof of Theorem 2.1, we need a general "connectedness lemma".

Lemma 2.3. Let $Q=\oplus_{n \geq 0} Q_{n}$ be a graded catenary equidimensional ring, assume that $\left(Q_{0}, m_{0}\right)$ is a local ring with infinite residue class field, and write $M=m_{0} \oplus Q_{+}$. Let $p, q, q^{\prime}, J$ be homogeneous $Q$-ideals of the same height $g$, with $p \supset J \subset q$ and $\sqrt{q}=\sqrt{q^{\prime}}$, and assume that $q^{\prime}$ is generated by homogeneous elements of the same degree. Further suppose that $Q / J$ satisfies Serre's condition $\left(S_{2}\right)$ and that $J_{x}$ is a prime ideal for every homogeneous element $x \in q^{\prime} \backslash M q^{\prime}$. Then ht $(p+q) \leq g+1$.

Proof. We consider the following subsets of the prime spectrum of $Q$ :

$$
\begin{aligned}
A & =\{\text { prime ideals of height } g \text { containing } J\} \\
& =\{\text { minimal prime ideals of } J\} \\
B & =\{\text { prime ideals of height } g \text { containing } p\} \neq \varnothing,
\end{aligned}
$$




$$
\begin{aligned}
C & =\{\text { prime ideals of height } g \text { containing } q\} \\
& \left.=\text { prime ideals of height } g \text { containing } q^{\prime}\right\} .
\end{aligned}
$$

If $B \cap C \neq \varnothing$, then ht $(p+q)=g$. Thus we may assume $B \cap C=\varnothing$, and since $B \cup C \subset A$ it follows that $B \subset A \backslash C$. Now $q^{\prime} \not \subset \cup_{P \in A \backslash C} P$, and $q^{\prime}$ is generated by homogeneous elements of the same degree. Thus there exists a homogeneous element $x \in q^{\prime} \backslash M q^{\prime}$ with $x \notin \bigcup_{P \in A \backslash C} P$. Then by our assumption, $J_{x}$ is prime, and since $x \notin \bigcup_{P \in A \backslash C} P$ it follows that $A \backslash C$ consists of at most one element. But on the other hand, $\varnothing \neq B \subset A \backslash C$, and hence $B=A \backslash C$. Therefore $B \cup C=A$ which implies that $\sqrt{p q}=\sqrt{J}$. But then by Hartshorne's connectedness theorem ([4]), grade $(p+q) / J \leq 1$, and hence ht $(p+q) / J \leq 1$ since $Q / J$ satisfies $\left(S_{2}\right)$. Therefore ht $(p+q)$ $\leq g+1$ because $Q$ is equidimensional and catenary.

Proof of Theorem 2.1. We may assume that the residue class field of $S$ is infinite. Choose $w$ as in Proposition 2.2, then $S\left[I t, I^{(2)} t^{2}\right]=S\left[I t, w t^{2}\right]$. In addition to the notations introduced prior to Proposition 2.2, write $D=S\left[I t, I^{(2)} t^{2}\right], Q=P[W]=S\left[T_{1}, T_{2}, T_{3}, W\right]$ where $W$ is a variable, and define an epimorphism $\varepsilon: Q \rightarrow D$ that extends $\varphi$ and maps $W$ to $w t^{2}$. In $Q$ consider the ideals $p=\operatorname{ker} \varepsilon, q=m Q$, and $J=\left(F_{1}, F_{2}, G_{1}, G_{2}, G_{3}\right)$ where $G_{i}=x_{i} W-\Delta_{i}$. First notice that $J \subset p$, since $\left(F_{1}, F_{2}\right) P=\operatorname{ker} \varphi$ and $\varepsilon\left(G_{i}\right)$ $=x_{i} w t^{2}-D_{i} t^{2}=0$ by Proposition 2.2. On the other hand, $\left(F_{1}, F_{2}\right) Q=$ $(\operatorname{ker} \varphi) Q$ is a prime ideal of height 2 with $G_{i} \notin\left(F_{1}, F_{2}\right) Q$, and therefore ht $J \geq 3$. Since ht $p=3$ it follows that ht $J=3$. Thus $p, q$, $J$ are homogeneous ideals of the same height 3 .

Next we show that $J$ is a Gorenstein ideal. To this end let $X_{1}, X_{2}$, $X_{3}$ be variables over $Q$, let $Y$ be a generic 2 by 3 matrix, let $\tilde{\Delta}_{1}, \tilde{\Delta}_{2}, \tilde{\Delta}_{3}$ be the maximal minors of $Y$ (with appropriate signs), write $\tilde{Q}=Q[Y] \otimes_{S}$ $S\left[X_{1}, X_{2}, X_{3}\right]_{\left(m, X_{1}, X_{2}, X_{3}\right)}$, and let $\tilde{J}$ be the $\tilde{Q}$-ideal generated by the elements $\sum_{j=1}^{3} Y_{i j} X_{j}, 1 \leq i \leq 2$, and $X_{i} W-\tilde{\Delta}_{i}, 1 \leq i \leq 3$. Then $\tilde{J}$ is a homogeneous Gorenstein ideal of height 3 ([6]). If we map $X_{i}$ to $x_{i}$ and $Y_{i j}$ to $\gamma_{i j}$, then $\tilde{J}$ specializes to $J$, and since $\tilde{J}$ is Cohen-Macaulay and ht $\tilde{J}=\mathrm{ht} J$, it follows that the kernel of this specialization is generated by a regular sequence on $\tilde{Q} / \tilde{J}$. Thus also $J$ is a Gorenstein ideal.

Now we need to prove that the ideal $J$ is independent of the chosen generating set $x_{1}, x_{2}, x_{3}$ of $I_{1}(A)$ as along as we replace $x_{1}, x_{2}, x_{3}$ by $x_{1}^{\prime}, x_{2}^{\prime}$, $x_{3}^{\prime}$, where 


$$
\left(\begin{array}{l}
x_{1}^{\prime} \\
x_{2}^{\prime} \\
x_{3}^{\prime}
\end{array}\right)=E\left(\begin{array}{l}
x_{1} \\
x_{2} \\
x_{3}
\end{array}\right)
$$

and $E$ is a 3 by 3 matrix over $S$ with $\operatorname{det} E=1$.

To show this claim, let $C_{1}, C_{2}$ be 3 by 3 matrices over $S$ with

$$
\left(\begin{array}{l}
a_{i 1} \\
a_{i 2} \\
a_{i 3}
\end{array}\right)=C_{i}\left(\begin{array}{l}
x_{1} \\
x_{2} \\
x_{3}
\end{array}\right)
$$

Notice that

$$
\left(\begin{array}{l}
\gamma_{i 1} \\
\gamma_{i 2} \\
\gamma_{i 3}
\end{array}\right)=C_{i}^{*}\left(\begin{array}{l}
T_{1} \\
T_{2} \\
T_{3}
\end{array}\right)
$$

where "*" denotes transpose. Now define $\Gamma^{\prime}=\Gamma E^{-1}$, then

$$
\left(\begin{array}{l}
\gamma_{i 1}^{\prime} \\
\gamma_{i 2}^{\prime} \\
\gamma_{i 3}^{\prime}
\end{array}\right)=\left(E^{-1}\right) *\left(\begin{array}{l}
\gamma_{i 1} \\
\gamma_{i 2} \\
\gamma_{i 3}
\end{array}\right)=\left(E^{-1}\right) * C_{i}^{*}\left(\begin{array}{l}
T_{1} \\
T_{2} \\
T_{3}
\end{array}\right)=\left(C_{i} E^{-1}\right)^{*}\left(\begin{array}{l}
T_{1} \\
T_{2} \\
T_{3}
\end{array}\right)
$$

and

$$
\left(\begin{array}{l}
a_{i 1} \\
a_{i 2} \\
a_{i 3}
\end{array}\right)=\left(C_{i} E^{-1}\right)\left(\begin{array}{l}
x_{1}^{\prime} \\
x_{2}^{\prime} \\
x_{3}^{\prime}
\end{array}\right)
$$

Thus we may take $\Gamma^{\prime}=\Gamma E^{-1}$ to be the $\Gamma$-matrix associated to the new generators $x_{1}^{\prime}, x_{2}^{\prime}, x_{3}^{\prime}$ of $I_{1}(A)$. Let $\Delta_{1}^{\prime}, \Delta_{2}^{\prime}, \Delta_{3}^{\prime}$ be the maximal minors of $\Gamma^{\prime}$ (with appropriate signs), then

$$
\left(\begin{array}{l}
\Delta_{1}^{\prime} \\
\Delta_{2}^{\prime} \\
\Delta_{3}^{\prime}
\end{array}\right)=E\left(\begin{array}{l}
\Delta_{1} \\
\Delta_{2} \\
\Delta_{3}
\end{array}\right)
$$

and hence

$$
\left(\begin{array}{l}
x_{1}^{\prime} W-\Delta_{1}^{\prime} \\
x_{2}^{\prime} W-\Delta_{2}^{\prime} \\
x_{3}^{\prime} W-\Delta_{3}^{\prime}
\end{array}\right)=E\left(\begin{array}{l}
x_{1} W-\Delta_{1} \\
x_{2} W-\Delta_{2} \\
x_{3} W-\Delta_{3}
\end{array}\right)
$$

Therefore $x_{1}^{\prime}, x_{2}^{\prime}, x_{3}^{\prime}$ define the same ideal $J$ as $x_{1}, x_{2}, x_{3}$.

Next we prove that for every element $x \in I_{1}(A) \backslash m I_{1}(A)$, the localization $J_{x}$ is a prime ideal in $Q_{x}$. By the above, we may assume that $x=x_{1}$. But then $J_{x_{1}}$ contains the ideal $\left(F_{1}, F_{2}, W-x_{1}^{-1} \Delta_{1}\right) Q_{x_{1}}$, which is a prime ideal of height 3 since $Q_{x_{1}} /\left(F_{1}, F_{2}, W-x_{1}^{-1} \Delta_{1}\right) Q_{x_{1}} \cong B_{x_{1}}$. Therefore $J_{x_{1}}$ itself is prime. 
Now we are ready to complete the proof of Theorem 2.1. By Vasconcelos ([25]) or Schenzel ([18]) we know that $S(I)=D$ if and only if grade $m D \geq 2$. Since $D \cong Q / p$ and $q=m Q$, this inequality is equivalent to $\operatorname{grade}(p+q) / p \geq 2$.

We first consider the case where $J=0$. Then $J \subset\left(m, \Delta_{1}, \Delta_{2}, \Delta_{3}\right) Q \subset$ $m Q=q . \quad$ Write $q^{\prime}=I_{1}(A) Q$, and $M=m \oplus Q_{+}$. Now $p, q, q^{\prime}, J$ are homogeneous ideals of height 3 , with $p \supset J \subset q$ and $\sqrt{q}=\sqrt{q^{\prime}}$, and $q^{\prime}$ is generated by homogeneous elements of degree 0 . Moreover we have seen that $Q / J$ is Cohen-Macaulay and that $J_{x}$ is a prime ideal for every $x \in$ $I_{1}(A) \backslash m I_{1}(A)$ and hence for every homogeneous element $x \in q^{\prime} \backslash M q^{\prime}$. Therefore by Lemma 2.3 , ht $(p+q) \leq 4$. Thus grade $(p+q) / p \leq \operatorname{ht}(p+q) / p$ $\leq 1$, and hence $S(I) \neq D$.

Next we consider the case where $\lrcorner \neq 0$ (this part of the proof follows [25] very closely). Then $J \not \subset m Q=q$.

We first claim that $p=J$. Suppose that $I_{1}(A) \backslash m I_{1}(A)$ is contained in the union of all associated primes of $J$, then for some $K \in \operatorname{Ass}(J)$, $I_{1}(A) Q \subset K$ and hence $q=\sqrt{I_{1}(A) Q \subset K}$. But $q$ is a prime of height 3 and $J$ is a Cohen-Macaulay ideal of height 3. Therefore $q=K \supset J$, which is ruled out by our assumption. Thus there exists an element $x \in$ $I_{1}(A) \backslash m I_{1}(A)$ such that $x$ is regular on $Q / J$. Moreover by what we have seen before, $J_{x}$ is prime. Therefore also $J$ is prime and hence $p=J$ since ht $p=$ ht $J$.

In particular $D \cong Q / p=Q / J$ is Cohen-Macaulay, and then grade $m D \geq 2$ if and only if $\operatorname{dim} D / m D \leq 2$. However, $D / m D \cong Q /(p+q)=$ $Q /(q+J)=Q /\left(m Q, \Delta_{1}, \Delta_{2}, \Delta_{3}\right)=\left(S / m\left[T_{1}, T_{2}, T_{3}\right] / J\right)[W]$. Therefore $\operatorname{dim} D / m D$ $\leq 2$ if and only if ht $J \geq 2$ or, equivalently, ht $J=2$.

The proof of Theorem 2.1 also shows the following: Suppose that Assumption 1.6 is satisfied. If $I$ is self-linked and hence $J \neq 0$ (Proposition 1.8), then $S\left[I t, I^{(2)} t^{2}\right] \cong Q / J$ is Gorenstein (c.f. also [25]). If however $I$ is not self-linked and hence $J=0$, then $J \subset p \cap q$ (using the notations of the proof of Theorem 2.1), $p \neq q$, Ass $(J)=\{p, q\}$ (proof of Lemma 2.3), and $J_{p}=p J$ (proof of Theorem 2.1). Therefore $J=K \cap p$ where $K$ is the $q$-primary component of $J$, or in other words, the defining ideal $p$ of $S\left[I t, I^{(2)} t^{2}\right]$ is geometrically linked to $K$ with respect to the Gorenstein ideal $J$ (for the definition of geometric linkage see [14]). (In fact, now the main step in the proof of Theorem 2.1 for $\bar{A}=0$ can be recast in the 
statement that up to radical, $p+q / p$ is a canonical ideal of $S\left[I t, I^{(2)} t^{2}\right]$.) In many instances one can compute $K$ and it turns out that $S\left[I t, I^{(2)} t^{2}\right]$ need not be Gorenstein as will be shown in the next example.

Example 2.4. Consider the monomial curve singularity $R=k \llbracket X, Y$, $Z \rrbracket / I=k \llbracket t^{7}, t^{9}, t^{10} \rrbracket$. Now

$$
A=\left(\begin{array}{lll}
X^{3} & Y^{2} & Z^{2} \\
Z & X & Y
\end{array}\right)
$$

and $J=\left(X^{3} T_{1}+Y^{2} T_{2}+Z^{2} T_{3}, Z T_{1}+X T_{2}+Y T_{3}, X W-Y T_{1} T_{2}+Z T_{3}^{2}, \quad Y W\right.$ $\left.-Z T_{1} T_{3}+X^{2} T_{1}^{2}, Z W-X^{2} T_{1} T_{3}-Y T_{2}^{2}\right)$. Then $J Q_{q}=(X, Y, Z) Q_{q}=q Q_{q}$, and hence $J=p \cap q$. Therefore $p$ and $q$ are linked with respect to $J$. Since $q=(X, Y, Z) Q$ is Cohen-Macaulay it follows by [14] that $S\left[I t, I^{(2)} t^{2}\right]$ $=Q / p$ is Cohen-Macaulay and that $r\left(S\left[I t, I^{(2)} t^{2}\right]\right)=\mu(q / J)=3$.

The next corollary is an immediate consequence of Theorem 2.1 and Proposition 1.8.

CoRollary 2.5. If under the Assumption 1.6, $S(I)=S\left[I t, I^{(2)} t^{2}\right]$, then $I$ is self-linked.

Thus when dealing with the question whether $S(I)-S\left[I t, I^{(2)} t^{2}\right]$, we may assume that $I$ is self-linked. But then by Proposition 1.8 one may suppose that $A=\left(\begin{array}{lll}x_{1} & x_{2} & a_{13} \\ x_{3} & a_{22} & a_{23}\end{array}\right)$ with $I_{1}(A)=\left(x_{1}, x_{2}, x_{3}\right)$ and $a_{13}=c x_{3}, a_{22}=$ $d_{1} x_{1}+d_{2} x_{2}+d_{3} x_{3}, a_{23}=e_{1} x_{1}+e_{2} x_{2}+e_{3} x_{3}$. In the following corollary we formulate the height condition of Theorem 2.1 in a more accessible way.

Corollary 2.6. With the above notations the following are equivalent:

a) $S(I)=S\left[I t, I^{(2)} t^{2}\right]$

b) either

$c \notin m$ and $\left(e_{2}, d_{1}+d_{2} d_{3}, e_{1}+d_{2} e_{3}-c d_{2}^{2}\right) \not \subset m$

or

$c \in m$ and $\left(e_{2} e_{3}, d_{1}+d_{2} d_{3}, e_{1}+d_{2} e_{3}+d_{3} e_{2}\right) \not \subset m$.

Proof. Write $k\left[T_{1}, T_{2}, T_{3}\right]=S / m\left[T_{1}, T_{2}, T_{3}\right]$, and let "-" denote reduction modulo $m$. Then

$$
\widetilde{\Gamma}=\left(\begin{array}{ccc}
T_{1} & T_{2} & \bar{c} T_{3} \\
l_{2} & l_{2} & l_{3}
\end{array}\right)
$$

where $l_{1}=\bar{d}_{1} T_{2}+\bar{e}_{1} T_{3}, l_{2}=\bar{d}_{2} T_{2}+\bar{e}_{2} T_{3}, l_{3}=T_{1}+\bar{d}_{3} T_{2}+\bar{e}_{3} T_{3}$. By Theorem 2.1 it suffices to show that the $k\left[T_{1}, T_{2}, T_{3}\right]$-ideal $\bar{\Delta}=I_{2}(\bar{\Gamma})$ has height two 
if and only if condition $2.6 \mathrm{~b}$ ) is satisfied.

We first consider the case where $c \notin m$. If $\bar{e} \neq 0$, then one can see from the Jacobi matrix that $k\left[T_{1}, T_{2}, T_{3}\right] /\left(\bar{\Delta}_{1}\right)$ is normal, and hence $\bar{\Delta}_{1}$ is a prime element. Moreover, $\bar{\Delta}_{1}$ does not divide $\bar{\Delta}_{3}$, and thus $h t(\bar{\Delta})=2$ in this case. If $\bar{e}_{2}=0$, then after subtracting $\bar{d}_{2}$ times the first row of $\bar{T}$ from the second row we may assume that

$$
\bar{\Gamma}=\left(\begin{array}{llll}
T_{1} & T_{2} & \bar{c} T_{3} \\
l_{1}^{\prime} & 0 & l_{3}^{\prime}
\end{array}\right)
$$

with $l_{1}^{\prime}=-\bar{d}_{2} T_{1}+\bar{d}_{1} T_{2}+\bar{e}_{1} T_{3}, \quad l_{3}^{\prime}=T_{1}+\bar{d}_{3} T_{2}+\left(\bar{e}_{3}-\bar{c} \bar{d}_{2}\right) T_{3} . \quad$ Now $\bar{\Delta}=$ $T_{2}\left(l_{1}^{\prime}, l_{3}^{\prime}\right)+\left(\bar{\Delta}_{2}\right)$, where $T_{2}$ does not divide $\bar{\Delta}_{2}$ and $\bar{\Delta}_{2} \in\left(l_{1}^{\prime}, l_{3}^{\prime}\right)$. Thus ht $\bar{\Delta}=2$ if and only if $l_{1}^{\prime}$ and $l_{3}^{\prime}$ are linearly independent. This in turn is equivalent to $\bar{d}_{1}+\bar{d}_{2} \bar{d}_{3} \neq 0$ or $\bar{e}_{1}+\bar{d}_{2} \bar{e}_{3}-\bar{c} \bar{d}_{2}^{2} \neq 0$.

Now we consider the case where $c \in m$. Then $\left(\bar{\Delta}_{1}, \bar{\Delta}_{2}\right)=\left(T_{1}, T_{2}\right) l_{3}$, with $l_{3}$ a prime element. Thus ht $\bar{\Delta}=2$ if and only if $l_{3}$ does not divide $\bar{\Delta}_{3}$. Writing $T_{1}=l_{3}-\bar{d}_{3} T_{2}-\bar{e}_{3} T_{3}$ we see that modulo $\left(l_{3}\right),-\bar{\Delta}_{3} \equiv\left(\bar{d}_{1}+\bar{d}_{2} \bar{d}_{3}\right) T_{2}^{2}$ $+\left(\bar{e}_{1}+\bar{d}_{2} \bar{e}_{3}+\bar{d}_{3} \bar{e}_{2}\right) T_{2} T_{3}+\bar{e}_{2} \bar{e}_{3} T_{3}^{2}$. Since $k\left[l_{3}, T_{2}, T_{3}\right]=k\left[T_{1}, T_{2}, T_{3}\right]$ it follows that $\bar{d}_{3} \neq \equiv 0 \bmod \left(l_{3}\right)$ if and only if $\bar{d}_{1}+\bar{d}_{2} \bar{d}_{3} \neq 0$, or $\bar{e}_{1}+\bar{d}_{2} \bar{e}_{3}+\bar{d}_{3} \bar{e}_{2} \neq 0$, or $\bar{e}_{2} \bar{e}_{3} \neq 0$.

Now let $A$ be a matrix as in Corollary 2.6. After multiplying $A$ from the right by the invertible 3 by 3 matrix

$$
\left(\begin{array}{ccc}
-c-d_{3} & d_{1} & 1 \\
1 & d_{2} & 0 \\
0 & -1 & 0
\end{array}\right)
$$

we may assume that

$$
A=\left(\begin{array}{ccc}
x_{2}-c x_{1}-d_{3} x_{1} & y & x_{1} \\
y & * & x_{3}
\end{array}\right)
$$

with $y=d_{1} x_{1}+d_{2} x_{2}-c x_{3}$ and $I_{1}(A)=\left(x_{1}, x_{2}, x_{3}\right)=\left(x_{2}-c x_{1}-d_{3} x_{1}, x_{1}, x_{3}\right)$. Thus instead of supposing that Assumption 2.6 is satisfied and $I$ is selflinked we may assume the following (see also Theorem 1.1 and Proposition 1.8):

Assumption 2.7. Suppose that

$$
A=\left(\begin{array}{lll}
x & y & u \\
u & z & v
\end{array}\right) \quad \text { with } I_{1}(A)=(x, u, v)
$$

write $y=a_{1} x+a_{2} u+a_{3} v, z=b_{1} x+b_{2} u+b_{3} v$, and let $f_{1}, f_{2}, f_{3}$ be the maximal minors of $A$ (with appropriate signs). 
In the remainder of this section we are interested in further describing a generator $w$ of $I^{(2)} / I^{2}$ (under the Assumption 2.7). We will write $w$ explicitly as a linear combination of the two elements $f_{3}, u f_{1}+v f_{3}$, which form a "self-linking" sequence of $I$ (Proposition 2.8). We then characterize those ideals $I$ for which $w$ can be chosen to be part of a self-linking sequence $f_{3}, w$ (Corollary 2.10), and prove that for such ideals necessarily $S(I)=S\left[I t, I^{(2)} t^{2}\right]$ (Proposition 2.11).

Proposition 2.8. Suppose that Assumption 2.7 is satisfied, let $w$ be as in Proposition 2.2, and set $a=\left(a_{1} b_{3}-a_{3} b_{1}\right) u-\left(a_{2}+b_{3}\right) v-a_{1} y+z$, $b=a_{3} b_{2}-a_{2} b_{3}$. Then

a) $w=a f_{3}+b\left(u f_{1}+v f_{2}\right)$

b) $I^{(2)} \subset\left(f_{3}, u f_{1}+v f_{2}\right)$.

Proof. We first prove part a). Choosing $x, u, v$ as a generating sequence of $I_{1}(A)$ we obtain

$$
\Gamma=\left(\begin{array}{ccc}
T_{1}+a_{1} T_{2} & a_{2} T_{2}+T_{3} & a_{3} T_{2} \\
a_{1} T_{1}+b_{1} T_{2} & a_{2} T_{1}+b_{2} T_{2} & a_{3} T_{1}+b_{3} T_{2}+T_{3}
\end{array}\right)
$$

Then

$$
w=\frac{D_{1}}{x}\left(a_{2} f_{2}+f_{3}\right)\left(a_{3} f_{1}+b_{3} f_{2}+f_{3}\right) x^{-1}-a_{3} f_{2}\left(a_{2} f_{1}+b_{2} f_{2}\right) x^{-1}
$$

Now our claim follows by an elementary calculation using the equations $f_{1}=y v-z u, f_{2}=y u-x v, f_{3}=x z-y^{2}$.

To prove part b), notice that by Proposition 2.2, $I^{(2)}=\left(I^{2}, w\right)$. Moreover $I^{2} \subset\left(f_{3}, u f_{1}+v f_{2}\right)$ by Remark 1.3 , and $w \in\left(f_{3}, u f_{1}+v f_{2}\right)$ by part a).

Before we can proceed we need a lemma.

Lemma 2.9. Suppose that Assumption 2.7 is satisfied and let $l=c_{1} f_{3}+$ $c_{2}\left(u f_{1}+v f_{2}\right)+h$ with $c_{i} \in S$ and $h \in I^{2}$. Then $\left(f_{3}, l\right)=\left(f_{3}, u f_{1}+v f_{2}\right)$ if and only if $c_{2}$ is a unit.

Proof. As $h \in I^{2}$ and $I^{2} \subset\left(f_{3}, u f_{1}+v f_{2}\right)$ by Remark 1.3 or Proposition 2.8 , there exist $d_{1}, d_{2}$ is $S$ such that $h=d_{1} f_{3}+d_{2}\left(u f_{1}+v f_{2}\right)$. Hence $\left(f_{3}, l\right)$ $=\left(f_{3},\left(c_{2}+d_{2}\right)\left(u f_{1}+v f_{2}\right)\right)$, and this ideal equals $\left(f_{3}, u f_{1}+v f_{2}\right)$ if and only if $c_{2}+d_{2}$ is a unit.

The assertion will follow once we have shown that $d_{2} \in m$. Suppose that $d_{2} \notin m$, then $u f_{1}+v f_{2}+g f_{3} \in I^{2}$, where $g=d_{1} d_{2}^{-1}$. The exact sequence 


$$
R^{2} \stackrel{A}{\longrightarrow} R^{3} \longrightarrow I / I^{2} \longrightarrow 0
$$

implies that modulo $I$,

$$
\left(\begin{array}{l}
u \\
v \\
g
\end{array}\right) \equiv s_{1}\left(\begin{array}{l}
x \\
y \\
u
\end{array}\right)+s_{2}\left(\begin{array}{l}
y \\
z \\
v
\end{array}\right) .
$$

Therefore $u-s_{1} x-s_{2} y \in I$ and $v-s_{1} y-s_{2} z \in I$. In other words, we may change $A=\left(\begin{array}{lll}x & y & u \\ y & z & v\end{array}\right)$ to $A^{\prime}=\left(\begin{array}{lll}x & y & u^{\prime} \\ y & z & v^{\prime}\end{array}\right)$ with $u^{\prime}$ and $v^{\prime}$ in $I$. Since $I=$ $I_{2}\left(A^{\prime}\right)$ it follows that $I=\left(x z-y^{2}\right)+m I$, which by Nakayama's Lemma would imply that $I$ is principal.

Corollary 2.10. Suppose that Assumption 2.7 is satisfied and let $w$ be any element such that $I^{(2)}=\left(I^{2}, w\right)$. Then the following are equivalent:

a) $I=\left(f_{3}, w\right): I$

b) $a_{3} b_{2}-a_{2} b_{3} \notin m$.

Proof. We first show that a) is equivalent to $\left(f_{3}, w\right)=\left(f_{3}, u f_{1}+v f_{2}\right)$. The latter equality certainly implies a), since $I=\left(f_{3}, u f_{1}+v f_{2}\right): I$ by Remark 1.3. Conversely assume a). Then the canonical module $\omega$ of $S / I$ can be computed as $\omega \cong\left(\left(f_{3}, w\right): I\right) /\left(f_{3}, w\right)=I /\left(f_{3}, w\right)$ ([14]). On the other hand, $\omega \cong\left(\left(f_{3}, u f_{1}+v f_{2}\right): I\right) /\left(f_{3}, u f_{1}+v f_{2}\right) \cong I /\left(f_{3}, u f_{1}+v f_{3}\right)$, and therefore $I /\left(f_{3}, w\right) \cong I /\left(f_{3}, u f_{1}+v f_{2}\right)$. By Proposition 2.8, $I^{(2)} \subset\left(f_{3}, u f_{1}+v f_{2}\right)$, hence $\left(f_{3}, w\right) \subset\left(f_{3}, u f_{1}+v f_{2}\right)$. Now the natural projection $I /\left(f_{3}, w\right) \rightarrow I /\left(f_{3}, u f_{1}+v f_{2}\right)$ is an epimorphism of isomorphic modules and therefore injective. Thus $\left(f_{3}, w\right)=\left(f_{3}, u f_{1}+v f_{2}\right)$.

Next we have to show that the equality $\left(f_{3}, w\right)=\left(f_{3}, u f_{1}+v f_{2}\right)$ is equivalent to b). By Proposition 2.8 we know that $w=\varepsilon\left(a f_{3}+b\left(u f_{1}+v f_{2}\right)\right)$ $+h$, where $\varepsilon$ is a unit, $b=a_{3} b_{2}-a_{2} b_{3}$, and $h \in I^{2}$. Now Lemma 2.9 implies that $\left(f_{3}, w\right)=\left(f_{3}, u f_{1}+v f_{2}\right)$ if and only if $a_{3} b_{2}-a_{2} b_{3} \notin m$.

Let us consider an example illustrating Proposition 2.8 and Corollary 2.10. We choose $A=\left(\begin{array}{lll}x & y & z \\ y & z & x\end{array}\right)$ with $I_{1}(A)=(x, y, z)$. Notice that $I=$ $I_{2}(I)$ is indeed a prime ideal if we assume for example that $S=\mathbb{R}[x, y, z]_{(x, y, z)}$. Now let $w=D_{1} / x$ be as in Proposition 2.2, then $I^{(2)}=\left(I^{2}, w\right)$. Moreover $w=\left(f_{2}^{2}-f_{1} f_{3}\right) x^{-1}=x^{3}+y^{3}+z^{3}-3 x y z=-z f_{1}-x f_{2}-y f_{3}$. On the other hand we may transform $A$ into $\left(\begin{array}{lll}x & y & u \\ y & z & v\end{array}\right)$ with $u=x+z$ and $v=x+y$. Then $I_{1}(A)=(x, u, v)$ and, using the notation of Corollary 2.10, $a_{3} b_{2}-a_{2} b_{3}$ 
$=1$. Now Corollary 2.10 implies that $I=\left(f_{3}, w\right): I$. Thus $I^{(2)}=\left(I^{2}\right.$, $\left.z f_{1}+x f_{2}+y f_{3}\right)$, and $I=\left(f_{3}, z f_{1}+x f_{2}+y f_{3}\right): I$.

Before we consider more examples we need the following result:

Proposition 2.11. Suppose that Assumption 1.6 is satisfied and that there exist elements $f \in I$ and $w \in I^{(2)}$ such that

i) $I^{(2)}=\left(I^{2}, w\right)$

ii) $I=(f, w): I$.

Then $S(I)=S\left[I t, I^{(2)} t^{2}\right]$.

Proof. Since $w \in I^{(2)} \subset m I$ (Proposition 2.8), and $I=(f, w): I$ it follows from Remark 1.4 that $f \notin m I$. In particular, $f \notin I^{(2)}$ and we may assume that $I=\left(f_{1}, f_{2}, f_{3}\right)$ with $f_{3}=f$. As $I^{2} \subset\left(f_{3}, w\right)$, we obtain equations $f_{1}^{2}=$ $g_{1} f_{3}+h_{1} w, f_{2}^{2}=g_{2} f_{3}+h_{2} w$. Here $g_{i} f_{3} \in I^{(2)}$, and thus $g_{i} \in I$ since $f_{3} \notin I^{(2)}$. Then we may write $g_{i}=c_{i 1} f_{1}+c_{i 2} f_{2}+c_{i 3} f_{3}$, and get the following relations:

$$
\begin{aligned}
& f_{1}^{2}-c_{11} f_{1} f_{3}-c_{12} f_{2} f_{3}-c_{13} f_{3}^{2}-h_{1} w=0 \\
& f_{2}^{2}-c_{21} f_{1} f_{3}-c_{22} f_{2} f_{3}-c_{23} f_{3}^{2}-h_{2} w=0 .
\end{aligned}
$$

Now present $D=S\left[I t, I^{(2)} t^{2}\right]=S\left[f_{1} t, f_{2} t, f_{3} t, w t^{2}\right]$ as a quotient of the polynomial ring $S\left[T_{1}, T_{2}, T_{3}, W\right]$ (see the proof of Theorem 2.1). Then because of the two relations (*), $D / m D$ is an epimorphic image of $S / m\left[T_{1}, T_{2}, T_{3}, W\right]$ $/\left(T_{1}^{2}+l_{1}, T_{2}^{2}+l_{2}\right)$ where $l_{i} \in\left(T_{3}, W\right)$. Therefore ht $m D \geq 2$, and hence grade $m D \geq 2$ since $D$ is Cohen-Macaulay by the remarks following the proof of Theorem 2.1. Now [25] or [18] implies that $D=S(I)$.

We now apply our theory to monomial space curves.

CoRollary 2.12. Let $R=S / I=k \llbracket t^{n_{1}}, t^{n_{2}}, t^{n_{3}} \rrbracket$ be a monomial space curve singularity that is not a complete intersection. As in Corollary 1.10 write

$$
A=\left(\begin{array}{lll}
X^{\alpha_{1}} & Y^{\beta_{1}} & Z^{\gamma_{1}} \\
Z^{\gamma_{2}} & X^{\alpha_{2}} & Y^{\beta_{2}}
\end{array}\right)
$$

Then the following are equivalent:

a) $S(I)=S\left[I t, I^{(2)} t^{2}\right]$

b) there exist elements $f \in I$ and $w \in I^{(2)}$ such that

i) $I^{(2)}=\left(I^{2}, w\right)$

ii) $I=(f, w): I$

c) $\alpha_{1}=\alpha_{2}$ and $\beta_{1} \leq \beta_{2}$ and $\gamma_{1} \geq \gamma_{2}$, or, $\alpha_{1} \geq \alpha_{2}$ and $\beta_{1}=\beta_{2}$ and $\gamma_{1} \leq \gamma_{2}$, or, 


$$
\alpha_{1} \leq \alpha_{2} \text { and } \beta_{1} \geq \beta_{2} \text { and } \gamma_{1}=\gamma_{2} \text {. }
$$

Proof. Set $\alpha=\alpha_{2}-\alpha_{1}, \beta=\beta_{2}-\beta_{1}, \gamma=\gamma_{2}-\gamma_{1}$. Assume a), then by Corollary 2.5, $I$ is self-linked. Thus by Corollary 1.10, we may assume that $\alpha \geq 0, \beta \geq 0, \gamma \leq 0$ (the other cases follow by suitable permutations of the rows and columns of $\mathrm{A})$. But then $I_{1}(A)=\left(X^{\alpha_{1}}, Y^{\beta_{1}}, Z^{\gamma_{2}}\right)$ and

$$
A=\left(\begin{array}{ccc}
X^{\alpha_{1}} & Y^{\beta_{1}} & Z^{-\gamma} Z^{\gamma_{2}} \\
Z^{\gamma_{2}} & X^{\alpha} X^{\alpha_{1}} & Y^{\beta} Y^{\beta_{1}}
\end{array}\right)
$$

Now Corollary 2.6 implies that if $\gamma=0$ then $\alpha=0$ or $\beta=0$, whereas if $-\gamma>0$ then $\alpha=0$. Thus $\alpha=0$ and $\beta \geq 0$ and $\gamma=0$, or $\alpha \geq 0$ and $\beta=0$ and $\gamma=0$, or $\alpha=0$ and $\beta \geq 0$ and $\gamma \leq 0$. In either case, condition $c$ ) is satisfied.

Next we prove that c) implies b). After a cyclic permutation of the variables we may assume that $\alpha=0$ and $\beta \geq 0$ and $\gamma \leq 0$. Then $I_{1}(A)=$ $\left(X^{\alpha_{1}}, Y^{\beta_{1}}, Z^{r_{2}}\right)$ and

$$
A=\left(\begin{array}{lll}
X^{\alpha_{1}} & Y^{\beta_{1}} & Z^{-r} Z^{\gamma_{2}} \\
Z^{\gamma_{2}} & X^{\alpha_{1}} & Y^{\beta} Y^{\beta_{1}}
\end{array}\right)
$$

We may transform $A$ into the form

$$
\left(\begin{array}{lll}
Y^{\beta_{1}} & X^{\alpha_{1}} & X^{\alpha_{1}}+Z^{-\gamma} Z^{\gamma_{2}} \\
X^{\alpha_{1}} & Z^{\gamma_{2}} & Z^{\gamma_{2}}+Y^{\beta} Y^{\beta_{1}}
\end{array}\right)
$$

This matrix has the form required in Assumption 2.7 since it is almost symmetric and $I_{1}(A)=\left(Y^{\beta_{1}}, X^{\alpha_{1}}+Z^{-\gamma} Z^{\gamma_{2}}, Z^{\gamma_{2}}+Y^{\beta} Y^{\beta_{1}}\right)$. Now write

$$
\begin{aligned}
& X^{\alpha_{1}}=Z^{-\gamma} Y^{\beta}\left(Y^{\beta_{1}}\right)+1\left(X^{\alpha_{1}}+Z^{-\gamma} Z^{\gamma_{2}}\right)-Z^{-\gamma}\left(Z^{\gamma_{2}}+Y^{\beta} Y^{\beta_{1}}\right) \\
& Z^{\gamma_{2}}=-Y^{\beta}\left(Y^{\beta_{1}}\right)+0\left(X^{\alpha_{1}}+Z^{-\gamma} Z^{\gamma_{2}}\right)+1\left(Z^{\gamma_{2}}+Y^{\beta} Y^{\beta_{1}}\right) .
\end{aligned}
$$

Then with the notations of Assumption 2.7, $a_{3} b_{2}-a_{2} b_{3}=1 \notin m$, and thus our assertion follows from Corollary 2.10.

Finally b) implies a) by Proposition 2.11.

The equivalence of a) and c) in Corollary 2.12 has been independently proven by T. Marley, and the fact that c) implies a) has been previously shown by P. Schenzel ([19]). We conclude this paper with an example which indicates that a) and b) in Corollary 2.12 might not be equivalent for general space curves.

Let $(T, n)$ be a three-dimensional regular local ring, let $x_{1}, x_{2}, x_{3}$ be an arbitrary system of parameters of $T$, and consider the $T$-ideal $K=I_{2}(B)$ where 


$$
B=\left(\begin{array}{llll}
x_{1} & x_{2} & x_{3} & 0 \\
0 & x_{1} & x_{2} & x_{3}^{2} \\
0 & 0 & x_{1} & x_{2}
\end{array}\right)
$$

Then $K$ is a perfect ideal of height 2 which is generically a complete intersection. Let $h_{1}, h_{2}, h_{3}, h_{4}$ be the maximal minors of $B$ (with appropriate signs), let $z_{i j}(1 \leq i \leq 2,1 \leq j \leq 2)$ be variables over $T$, set $S=T\left(z_{i j}\right)=$ $T\left[z_{i j}\right]_{n T\left[z_{i j}\right]}$, and $\alpha_{1}=h_{1}+z_{11} h_{3}+z_{21} h_{4}, \alpha_{2}=h_{2}+z_{12} h_{3}+z_{22} h_{4}$. Then $I=$ $\left(\alpha_{1}, \alpha_{2}\right) S: K S$ is an $S$-ideal of height 2. Moreover $I$ is a "universal link" of $K$ ([12]), and therefore $I$ is actually a prime ideal ([12]). On the other hand, notice that

$$
\begin{aligned}
I & =I_{4}\left(\begin{array}{ccccc}
x_{1} & 0 & 0 & 1 & 0 \\
x_{2} & x_{1} & 0 & 0 & 1 \\
x_{3} & x_{2} & x_{1} & z_{11} & z_{12} \\
0 & x_{3}^{2} & x_{2} & z_{21} & z_{22}
\end{array}\right) \\
& =I_{2}\left(\begin{array}{ccc}
x_{3}-z_{11} x_{1}-z_{12} x_{2} & x_{2}-z_{12} x_{1} & x_{1} \\
-z_{21} x_{1}-z_{22} x_{2} & x_{3}^{2}-z_{22} x_{1} & x_{2}
\end{array}\right) \\
& =I_{2}\left(\begin{array}{ccc}
x_{1} & x_{2} & x_{3}-z_{11} x_{1}-z_{12} x_{2} \\
x_{2} & x_{3}^{2}-z_{22} x_{1}+z_{12} x_{2} & -z_{21} x_{1}-z_{22} x_{2}
\end{array}\right) \\
& =I_{2}(A),
\end{aligned}
$$

where $A=\left(\begin{array}{lll}x & y & u \\ y & z & v\end{array}\right)$ with $x=x_{1}, u=x_{3}-z_{11} x_{1}-z_{12} x_{2}, v=-z_{21} x_{1}-z_{22} x_{2}$, $y=x_{2}, z=x_{3}^{2}-z_{22} x_{1}+z_{12} x_{2}$. Then $I_{1}(A)=(x, u, v)$, and writing $y=a_{1} x$ $+a_{2} u+a_{3} v, z=b_{1} x+b_{2} u+b_{3} v$, we get $a_{1} \equiv-z_{21} / z_{22} \bmod m, a_{2} \equiv 0 \bmod m$, $a_{3}=-1 / z_{22} \bmod m, b_{1} \equiv-z_{22}-z_{12} z_{21} / z_{22} \bmod m, b_{2} \equiv 0 \bmod m, b_{3} \equiv z_{12} / z_{22} \bmod m$. Thus $a_{3} b_{2}-a_{2} b_{3} \in m$, and now Proposition 2.11 implies that there exists no element $w$ such that $I^{(2)}=\left(I^{2}, w\right)$ and $I=\left(f_{3}, w\right): I$. On the other hand, one can show using Corollary 2.6 that $S(I)=S\left[I t, I^{(2)} t^{2}\right]$.

\section{REFERENCES}

[1] R. Cowsik, Symbolic powers and number of defining equations, in "Algebra and its applications (New Delhi, 1981)", Lecture Notes in Pure and Appl. Math., Vol. 91, Dekker, New York, 1984, 13-14.

[2] R. Cowsik and Nori, On the fibres of blowing-up, J. Indian Math. Soc., 40 (1976), 217-222.

[ 3 ] S. Eliahou, Symbolic powers of monomial curves, preprint.

[4] R. Hartshorne, Complete intersections and connectedness, Amer. J. Math., 84 (1962), 497-508.

[5] J. Herzog, Generators and relations of abelian semigroups and semigroups rings, Manuscripta Math., 3 (1970), 153-193.

[6] _ Certain complexes associated to a sequence and a matrix, Manuscripta 
Math., 12 (1974), 217-247.

[7] - Ein Cohen-Macaulay-Kriterium mit Anwendungen auf den Konormalenmodul und den Differentialmodul, Math. Z., 163 (1978), 149-162.

[8] —, A. Simis, and W. Vasconcelos, Koszul homology and blowing-up rings, in "Commutative algebra, Proc. Trento Conf.", eds.: S. Greco and G. Valla, Lecture Notes in Pure and Appl. Math., Vol. 84, Dekker, New York, 1983, 79-169.

[ 9 ] C. Huneke, On the finite generation of symbolic blow-ups, Math. Z., 179 (1982), 465-472.

[10] - The primary components of and integral closures of ideals in 3-dimensional regular local ring's, Math. Ann., 275 (1986), 617-635.

[11] - Hilbert functions and symbolic powers, Michigan Math. J., 34 (1987), 293318.

[12] C. Huneke and B. Ulrich, The structure of linkage, Annals of Math., 126 (1987), 277-334.

[13] M. Morales, Noetherian symbolic blow-up and examples in any dimension, preprint.

[14] C. Peskine and L. Szpiro, Liaison des variétés algébriques, Invent. Math., 26 (1974), 271-302.

[15] A. P. Rao, On self-linked curves, Duke Math. J., 49 (1982), 251-273.

[16] P. Roberts, A prime ideal in a polynomial ring whose symbolic blow-up is not Noetherian, Proc. Amer. Math. Soc., 94 (1985), 589-592.

[17] L. Robbiano and G. Valla, Free resolutions for special tangent cones, in "Commutative algebra, Proc. Trento Conf.”, eds.: S. Greco and G. Valla, Lecture Notes in Pure and Appl. Math., Vol. 84, Dekker, New York, 1984, 253-274.

[18] P. Schenzel, Filtrations and Noetherian symbolic blow-up rings, to appear in Proc. Amer. Math. Soc.

[19] — Examples of Noetherian symbolic blow-up rings, Rev. Roumaine Math. Pures Appl., 33 (1988), 4, 375-383.

[20] L. Szpiro, "Equations defining space curves", Tata Institute of Fundamental Research, Bombay, Springer, Berlin, New York, 1979.

[21] G. Valla, On determinantal ideals which are set-theoretic complete intersections, Comp. Math., 42 (1981), 3-11.

[22] —- On set-theoretic complete intersections, in "Complete Intersections, Acireale 1983", eds.: S. Greco and R. Strano, Lecture Notes in Mathematics, Vol. 1092, Springer, Berlin, New York, 1984, 85-101.

[23] W. Vasconcelos, On linear complete intersections, J. Algebra, 111 (1987), 306-315.

[24] - On the structure of certain ideal transforms, Math. Z., 198 (1988), 435-448.

[25] —- Symmetric algebras and factoriality, preprint.

\author{
Jürgen Herzog \\ Fachbereich Mathematik \\ Universität-Gesamthochschule-Essen \\ Universitätstrasse 3 \\ 4300 Essen \\ Germany
}

Bernd Ulrich

Department of Mathematics

Michigan State University

East Lansing, Michigan 48824

$U S A$ 